\title{
Hypocellular acute leukemia: study of clinical and hematological features
}

\author{
Shano Naseem • Tushar Sehgal • Narender Kumar • Neelam Varma • Reena Das • \\ Jasmina Ahluwalia $\cdot$ MUS Sachdeva $\cdot$ Prashant Sharma • Pankaj Malhotra • \\ Subhash Varma
}

Received: 7 June 2014 / Accepted: 29 August 2014 / Published online: 18 September 2014

(C) Springer-Verlag Berlin Heidelberg 2014

\begin{abstract}
Hypocellular acute leukemia was previously referred to as smoldering leukemia. This is currently defined as having $\geq 20 \%$ blasts in peripheral blood or bone marrow with cellularity of less than $20 \%$ in bone marrow biopsy at presentation. Hypocellular variants of acute myeloid leukemia (AML) are commoner than that of acute lymphoid leukemia (ALL). They may pose a diagnostic challenge to the treating clinician and pathologist as they can simulate hypoplastic myelodysplastic syndrome and aplastic anemia. It is of utmost importance to distinguish these overlapping disorders as treatment modalities differ. Details of clinical features, complete blood counts, bone marrow aspirate findings, percentage cellularity, pattern of infiltration in trephine biopsies, and immunophenotyping by flow cytometry or immunohistochemistry were analyzed in cases diagnosed as hypocellular acute leukemia. Hypocellular acute leukemia constituted $2.5 \%(8 / 316)$ of all diagnosed cases of acute leukemia during the study period. Seven of the eight cases of hypocellular acute leukemia were hypocellular AML while one was hypocellular ALL. Median age of patients was 45 years, with a male:female ratio of 1.7:1. Mean duration of symptoms was 1.2 months, with most common presenting feature being fever. None of the patients except one had an antecedent hematologic disorder or chemotherapy or radiotherapy. Hypocellular acute leukemia is important to recognize from other overlapping disorders. Clinical parameters and
\end{abstract}

S. Naseem $(\bowtie) \cdot$ T. Sehgal $\cdot$ N. Kumar $\cdot$ N. Varma $\cdot$ R. Das $\cdot$

J. Ahluwalia $\cdot$ M. Sachdeva $\cdot$ P. Sharma

Department of Hematology, Postgraduate Institute of Medical

Education and Research, Chandigarh 160012, India

e-mail: shanonaseem@yahoo.co.in

P. Malhotra $\cdot$ S. Varma

Department of Internal Medicine, Postgraduate Institute of Medical

Education and Research, Chandigarh, India laboratory data including immunophenotyping can aid in distinguishing them.

Keywords Acute leukemia $\cdot$ Bone marrow $\cdot$ Hypocellular

\section{Introduction}

Acute leukemias usually present with a hypercellular bone marrow. However, less than $10 \%$ of the cases may have a hypocellular bone marrow at presentation. Hypocellular acute leukemia (HAL) is currently defined as acute leukemia with a bone marrow cellularity $\leq 20 \%$, although in some reports, cellularity less than $40 \%$ is considered to be hypocellular [1-3]. Acute myeloid leukemia (AML) has been reported to be much more common than acute lymphoblastic leukemia (ALL) $[4,5]$. The incidence of hypocellular AML ranges between 5 and $12 \%$ of all cases of AML [1, 6]. They occur mainly in adults and are usually secondary in nature and may develop after radiation or chemotherapy [5]. By contrast, hypocellular ALL occurs predominantly in children and constitutes $2-3 \%$ of childhood ALL [7].

HAL needs to be differentiated from hypoplastic myelodysplastic syndrome (MDS) and aplastic anemia (AA), as these also have peripheral cytopenias and bone marrow hypocellularity. In addition, hypoplastic MDS cases may not have very evident dysplastic features because of low cellularity. Clinical parameters like duration of symptoms, history of antecedent hematologic disorder, or prior chemotherapy or radiotherapy may aid in diagnosis. Guidelines have been proposed to facilitate the differentiation between these disorders $[4,8]$.

No significant difference in the cytogenetic abnormalities between patients with hypocellular AML and those with nonhypocellular AML has been reported. However, molecular studies show a lower frequency of RAS and FLT3 mutations 
( 2 and $4 \%$, respectively) in patients with hypocellular AML which is similar to that seen in patients with MDS. Treatment options are similar to that of non-hypocellular AML. On multivariate analysis, overall survival, remission duration, and event-free survival have been reported to be comparable to that of non-hypocellular AML [9].

Most case series on HAL are from western countries [2, 4, $9,10]$. On literature search, no large study on HAL could be retrieved from India; reported HAL are restricted to case reports only $[11,12]$. We therefore planned this study to assess the frequency and clinical and hematological features of HAL in our group of acute leukemia patients.

\section{Materials and methods}

All cases reported as HAL from May 2012 to May 2013 were analyzed. Patient group comprised of freshly diagnosed cases of acute leukemia which showed hypocellularity (bone marrow cellularity $\leq 40 \%$ ) at presentation. Clinical data regarding age, clinical presentation, duration of illness, drug and treatment history, and laboratory data including complete blood count, peripheral smear, and bone marrow findings including cytochemistry for myeloperoxidase (MPO) and periodic acid Schiff (PAS) were noted. Trephine biopsies were assessed for percentage cellularity and pattern of infiltration. Quantification of bone marrow reticulin fibrosis was done using Bauermeister's grading system [13]. Immunophenotyping by flow cytometry or immunohistochemistry (IHC) was done to assess the lineage of blasts.

For flow cytometric immunophenotyping, samples $(1 \times$ $10^{6}$ cells/tube) were stained with adequate concentrations of monoclonal antibodies directed against cell surface markers using lyse-stain-wash technique. Initially, primary panel of monoclonal antibodies was put (CD13, CD33, CD117, MPO - for myeloid lineage; CD11c, CD14, CD64-for monocytic differentiation; CD10, CD19, CD20c, CD22c, CD79a-for B-lymphoid lineage; CD7, CD4, CD8c, CD3for T-lymphoid lineage; and CD34, TdT, and HLA-DR) followed by secondary panel (CD41, CD61-for megakaryocytic lineage; kappa and lambda in cases with B-lineage; and $\mathrm{CD} 2, \mathrm{CD} 5, \mathrm{CD} 3, \mathrm{CD} 1 \mathrm{a}$ in cases with T-lineage) based on the results of primary panel. Samples were acquired immediately after preparation using the FACS Canto II cytometer and the FACS Diva software program (BD Biosciences, USA). Blasts were gated on CD45 versus side scatter plot (dim CD45 and low side scatter), and a positive expression was defined when $20 \%$ or more blast cells showed fluorescence intensities in excess of the fluorescence intensity associated with negative control.

IHC was done on bone marrow trephine biopsy sections of thickness 3-4 $\mu \mathrm{m}$ using antibodies against CD34, MPO, TdT,
CD3, CD19, and CD20 in cases where flow cytometric immunophenotyping was inconclusive.

\section{Results}

In this study, we found eight cases of HAL which constituted $2.5 \%(8 / 316)$ of all diagnosed cases of acute leukemia. These cases were diagnosed as HAL based on a bone marrow cellularity $\leq 20 \%$ and blast count of $\geq 20 \%$ in bone marrow or peripheral blood. Two cases showed cellularity between 20 and $40 \%$; these were also included in the study, based on previous reports of HAL [1-3].

Seven $(87.5 \%)$ of these cases were diagnosed as hypocellular AML while one (12.5\%) case was diagnosed as hypocellular ALL. The incidence of hypocellular AML was $2.2 \%$ and of hypocellular ALL was $0.3 \%$ of all acute leukemia cases and $3.2 \%$ and $1 \%$ of AML and ALL cases, respectively.

Median age of patients with HAL was 45 years (range, 16 to 50 years). There was a male predominance; males constituted $63 \%$ of all cases (5/8) and female $37 \%(3 / 8)$. Clinically in four patients (50\%), AA or MDS was suspected because of absence of organomegaly, presence of cytopenias and no circulating blasts. All the cases presented with a short history of symptoms, mean duration being 1.2 months (range 12 months). Most common presenting complaint was fever seen in all patients (8/8); other presenting complaints were bleeding episodes (in the form of epistaxis and malena) and abdominal pain in one patient each. Splenomegaly and cervical lymphadenopathy were seen in one and two patients, respectively. Only one patient $1 / 8(12.5 \%)$ had an antecedent hematologic disorder, i.e., MDS-refractory anemia with excess blasts-1 (MDS-RAEB-1). There was no history of prior hematological disorder or therapy in remaining patients.

Median hemoglobin levels were $7.6 \mathrm{~g} / \mathrm{dL}$ (range, $4.8-11.1 \mathrm{~g} /$ $\mathrm{dL}$ ), median total leucocyte count (TLC) was $2.65 \times 10^{9} / \mathrm{L}$ (range, $1.5-5 \times 10^{9} / \mathrm{L}$ ), and median platelet count was $42.5 \times$ $10^{9} / \mathrm{L}$ (range, $4-391 \times 10^{9} / \mathrm{L}$ ). Platelet counts were preserved in only two patients. Five $(63 \%)$ patients had bicytopenia and three $(37 \%)$ had pancytopenia at presentation. Peripheral blood blasts were seen in six patients, and in remaining two, there were no circulating blasts.

Bone marrow cellularity was $\leq 20 \%$ in six patients (range, 10-20\%) and between $>20$ and $40 \%$ in two patients (Fig. 1). Reticulin fibrosis was increased in only one patient, and in remaining seven, it was not increased. Normal hematopoietic lineage cells were reduced. Percentage of blasts in bone marrow ranged from $22-91 \%$.

Lineage of blasts was myeloid in seven $(87.5 \%)$ cases and lymphoid in one case. Most common subtype was AML with minimal differentiation [French-American-British (FAB) AML-M0 and AML-M1] seen in three patients each followed 
Fig. 1 a Trephine biopsy section showing hypocellular bone marrow (Hematoxylin and Eosin, 400×). b High-power view showing interstitial excess of blasts (Hematoxylin and Eosin $1,000 \times)$

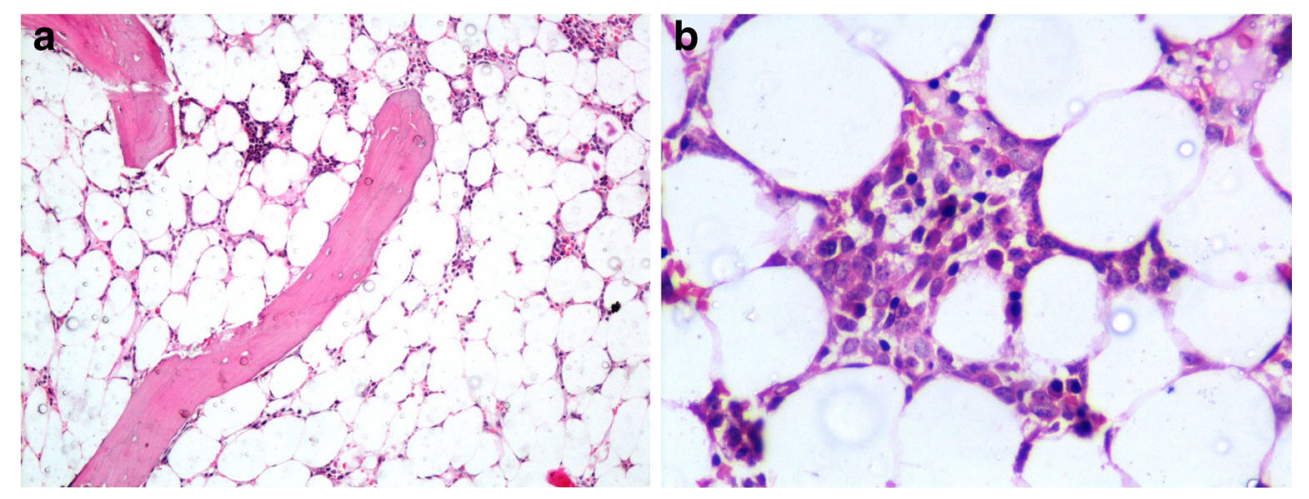

by AML with myelomonocytic differentiation (AML-M4) and T-ALL in one case each.

Of the eight patients, three patients underwent treatment and five did not opt for therapy due to low socio-economic status, financial constraints, or poor general condition. Of the three patients, two died during chemotherapy treatment, one during induction, and another during maintenance. The third patient underwent allogeneic bone marrow transplant after the 1 st induction therapy. However, he developed acute graft versus host disease 144 days post-transplant and was subsequently started on cyclosporine. He is doing well now and is on follow-up being day 250 post-transplant.

Clinical and hematological details of patients are outlined in Table 1 briefly.

\section{Discussion}

HAL is an uncommon entity, whose pathogenesis is still a mystery. In bonafide acute leukemia cases with hypercellular bone marrow, deficient normal hematopoiesis is explained by complete replacement of marrow by leukemic cells. However, that is not the case in acute leukemia patients with hypocellular bone marrow. Researchers have tested serum of patients of HAL and have shown inhibitory role of leukemic blasts on normal hematopoiesis. The inhibitory substances identified include leukemia inhibitor factor (LIF), stem cell inhibitor factor (SCI), tumor necrosis factor (TNF), prostaglandin E, and decreased or aberrant stimulatory factors such as granulocyte colony stimulating factor (G-CSF) [10].

On literature search, most HAL series are from western countries and no large series on HAL could be retrieved from India, except for few case reports $[11,12]$. In this study, we therefore investigated the incidence of HAL in acute leukemia cases and systematically analyzed their clinical and hematological features in our group of acute leukemia patients.

The incidence of HAL was $2.5 \%(8 / 316)$ of all acute leukemia cases, of which hypocellular AML was $2.2 \%$ (7/316) and hypocellular ALL was $0.3 \%(1 / 316)$. Of all AML cases, hypocellular AML constituted $3.2 \%$, and of all
ALL cases, hypocellular ALL constituted $1 \%$. The reported incidence of hypocellular AML from western studies ranges between 5 and $12 \%$ of all cases of AML $[1,6]$. Hypocellular ALL occurs mostly in children and constitutes $2-3 \%$ of childhood ALL [7].

The median age of presentation of HAL cases in our study was 45 years which was lower than that of the study by Nagai et al. [4], in which it was 67 years and by Al-Kali et al. [9], who reported it as 65 years. The lower age of presentation in our group of patients could be because of geographical and ethnic causes. The previous report of hypocellular AML from India has also described it in young patients [11]. This aspect may be studied further in larger cohort of patients.

HAL are usually secondary in nature and have been reported to develop after an antecedent hematologic malignancy like MDS, primary myelofibrosis (PMF), or therapy with radiation or chemotherapy $[9,14,15]$. In a series of 123 patients by AlKali et al., nearly half the cases of hypocellular AML were composed of secondary AML, either with an antecedent hematologic disorder or prior history of chemotherapy or radiotherapy. However, in our series, only one patient 1/8 (12.5\%) had an antecedent hematologic disorder, i.e., MDS-RAEB-1; in others, even after extensive interrogation, no history of previous chemotherapy or radiotherapy or hematologic disorder could be obtained.

None of our patients had splenomegaly, which is important in differentiation of de-novo HAL from leukemic transformation of PMF. Patients with leukemic transformation of PMF usually give preceding history of it, though some patients may initially present in blast phase. Differentiation of such cases from HAL is facilitated by presence of massive splenomegaly in PMF cases [16].

HAL also needs to be differentiated from hypoplastic MDS and AA, and guidelines have been proposed to aid the differentiation between these disorders $[4,8]$. Along with morphological examination of peripheral blood film and bone marrow for dysplasia, special stains for iron on bone marrow aspirate (for ring sideroblasts) and reticulin stain on bone marrow biopsy (for degree of fibrosis) must be done in all the cases. An increase in bone marrow reticulin may be seen in 


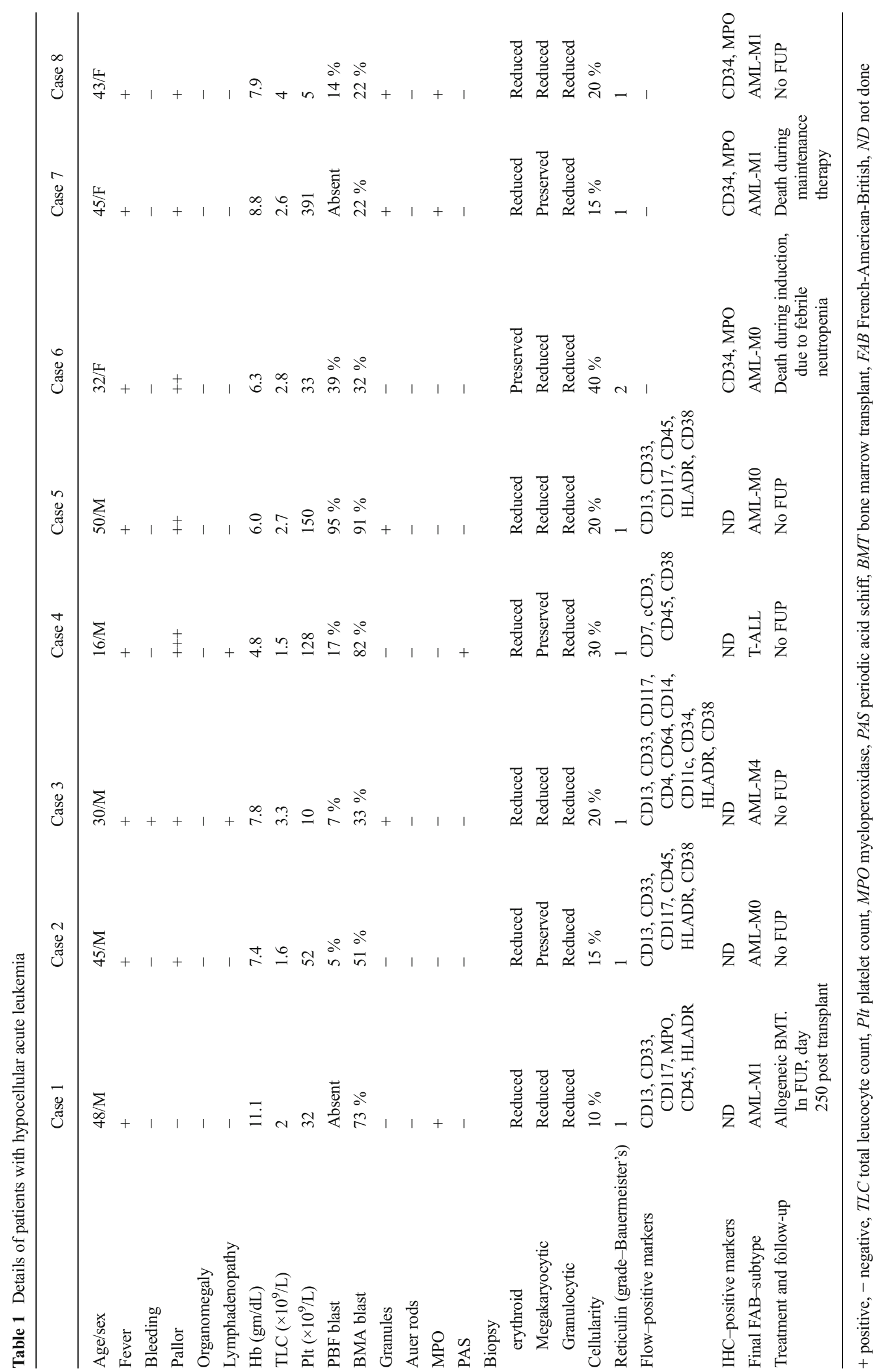


hypoplastic AML and hypoplastic MDS but is not increased in AA [17].

Previous studies have also shown increase in reticulin in hypocellular ALL [18, 19]; however, our single case of hypocellular ALL did not show increased reticulin, and overall also only one case had increased reticulin fibrosis.

Immunophenotype done using flow cytometry and IHC in our study showed AML in $88 \%$ cases among which AMLM0 and AML-M1 were the most common subtypes. Very few cases of hypocellular ALL have been reported in the literature; we also found one such case in this study. Hypocellular ALL usually presents in children but rare cases in adults have been documented [20]. Our patient of hypocellular ALL was a 16year-old boy, who presented with a history of fever and shortness of breath for 2 months. On physical examination, he had severe pallor with posterior cervical lymphadenopathy but no organomegaly. Hematologic examination revealed pancytopenia with hemoglobin of $4.8 \mathrm{~g} / \mathrm{dL}$, TLC of $1.5 \times$ $10^{9} / \mathrm{L}$, and platelet count of $128 \times 10^{9} / \mathrm{L}$. Peripheral blood film (PBF) revealed $17 \%$ blasts. Bone marrow aspirate smears were particulate, markedly hypocellular, and showed $82 \%$ blasts. Cytochemically, these blasts were MPO negative and PAS positive and on flow cytometry immunophenotyping, the lineage of blasts were T-lymphoid.

Although we could not perform genetic analysis in our cases, previous reports show that there is no difference in the cytogenetic abnormalities between patients with hypocellular AML versus non-hypocellular AML patients. There are also no specific molecular abnormalities defined; however, there is a lower frequency of RAS and FLT3 mutations ( 2 and $4 \%$, respectively) in patients with hypocellular AML which may be consistent with the finding that FLT3- and RAS-mutated myeloid malignancies are more proliferative in nature [9].

In our study, only three patients opted for treatment and two of these died during treatment. Unfortunately, our data on treatment details is limited to perform an outcome analysis. However, previous reports on HAL treatment and survival show that it has an indolent course and can commonly achieve a good response to remission induction therapy. Treatment options are similar to that of the patients with nonhypocellular AML. Complete remission (CR) rates in patients of hypocellular AML have been reported to be similar to that in patients with non-hypocellular AML. Also, overall survival, remission duration, and event-free survival have been found to be comparable to those of patients with nonhypocellular AML in a large study on 123 patients of hypocellular AML and 1,219 non-hypocellular AML [9].

Few studies have also shown beneficial effects of hematopoietic growth factors-G-CSF in the treatment of hypocellular AML [21-23]. The possible hypothesis which has been suggested for this response to G-CSF is that (i) in HAL, leukemic cells must have characteristics that sensitize them to G-CSF, (ii) HAL cells proliferate slowly and therefore characterized by a maturation failure and G-CSF efficaciously results in leukemic cell maturation, and (iii) benefit of G-CSF may be also be due to other biological characteristics, i.e., GCSF could suppress the leukemic cell clone by inducing apoptosis or cause increase in effector cytotoxic cells secondary to increased normal hematopoiesis [22]. It is proposed that G-CSF can be useful especially in some selected cases of AML especially in elderly patients with hypocellular AML who may not be likely candidates for chemotherapy.

\section{Conclusion}

To summarize, HAL is a challenging entity. It is generally seen in adults with hypocellular AML, being much commoner than hypocellular ALL. It is characterized by low bone marrow cellularity, prominent cytopenias, and in many cases, clinically they simulate AA and hypoplastic MDS. However, it is important to differentiate these conditions as treatment modalities differ. HAL usually has an indolent course and can commonly achieve a good response to remission induction therapy.

We would like to emphasize that although the incidence of HAL is low, it is not very uncommon and must always be kept in mind in hypocellular bone marrows in order to avoid misdiagnosis and mismanagement. A comprehensive multiparametric diagnostic approach including clinical features, morphology, cytochemistry, immunophenotyping, and genetic analysis is very important for its diagnosis.

Conflict of interest The authors declare that they have no conflict of interest.

\section{References}

1. Needleman SW, Burns CP, Dick FR et al (1981) Hypoplastic acute leukemia. Cancer 48:1410-1414

2. Berdeaux DH, Glasser L, Serokmann R et al (1986) Hypoplastic acute leukemia: review of 70 cases with multivariate regression analysis. Hematol Oncol 4:291-305

3. Tomonaga M (1995) Hypocellular acute leukemia. Rinsho Ketsueki 36:457-464

4. Nagai K, Kohno T, Chen YX et al (1996) Diagnostic criteria for hypocellular acute leukemia: a clinical entity distinct from overt acute leukemia and myelodysplastic syndrome. Leuk Res 20:563-574

5. Matloub YH, Brunning RD, Arthur DC et al (1993) Severe aplastic anemia preceding acute lymphoblastic leukemia. Cancer 71:264-268

6. Tuzuner N, Cox C, Rowe JM et al (1995) Hypocellular acute myeloid leukemia: the Rochester (New York) experience. Hematol Pathol 9: 195-203

7. Klingemann HG, Storb R, Sanders J et al (1986) Acute lymphoblastic leukaemia after bone marrow transplantation for aplastic anaemia. $\mathrm{Br}$ J Haematol 63:47-50

8. Bennett JM, Orazi A (2009) Diagnostic criteria to distinguish hypocellular acute myeloid leukemia from hypocellular 
myelodysplastic syndromes and aplastic anemia: recommendations for a standardized approach. Haematologica 94:264-268

9. Al-Kali A, Konoplev S, Lin E et al (2012) Hypocellular acute myeloid leukemia in adults: analysis of the clinical outcome of 123 patients. Haematologica 97:235-240

10. De-Bock R, de-Jonge M, Korthout M et al (1992) Hypoplastic acute leukemia: description of eight cases and search for hematopoietic inhibiting activity. Ann Hematol 65:247-252

11. Jain D, Singh T, Kumar N (2009) Hypocellular acute myeloid leukemia with bone marrow necrosis in young patients: two case reports. $\mathrm{J}$ Med Case Rep 3:27

12. Sinha S, Bhargava M (2013) Fanconi anemia presenting as an "evolving" acute leukemia-diagnostic challenges. Indian J Med Paediatr Oncol 34:305-308

13. Bain BJ, Clark DM, Wilkins BS (2010) Special techniques applicable to bone marrow diagnosis. In: Bone marrow pathology, 4th edn. Wiley-Blackwell, Oxford, pp 54-99

14. Fukushima T, Uchida M, Iwasaki $\mathrm{H}$, et al. Blastic crisis of primary myelofibrosis associated with multiple myeloblastomas Rinsho Ketsueki 1990;31:95-99.

15. Jurisic V, Colovic N, Pavlovic S et al (2007) Osteolytic lesions in leukemic transformation of myelofibrosis. Arch Oncol 15:45-47

16. Thiele J, Kvasnicka HM, Tefferi A et al (2008) Primary myelofibrosis. In: Swerdlow SH, Campo E, Harris NL et al (eds) WHO classification of tumours of haematopoietc and lymphoid tissues, 4th edn. IARC press, Lyon, pp 44-47

17. Bain BJ, Clark DM, Wilkins BS (2010) Infective and reactive changes. In: Bone marrow pathology, 4th edn. Wiley-Blackwell, Oxford, pp $110-165$

18. Reid MM, Summerfield GP (1992) Distinction between aleukaemic prodrome of childhood acute lymphoblastic leukaemia and aplastic anaemia. J Clin Pathol 45:697-700

19. Breatnach F, Chessells JM, Greaves MF (1981) The aplastic presentation of childhood leukaemia: a feature of common-ALL. Br J Haematol 49:387-393

20. Krober SM, Horny HP, Steinke B et al (2003) Adult hypocellular acute leukemia with lymphoid differentiation. Leuk Lymph 44:1797-1801

21. Lee M, Chubachi A, Niitsu H et al (1995) Successful hematopoietic reconstitution with granulocyte colony-stimulating factor in a patient with hypoplastic acute myelogenous leukemia. Intern Med 34:692694

22. Nimubona S, Grulois I, Bernard M et al (2002) Complete remission in hypoplastic acute myeloid leukemia induced by G-CSF without chemotherapy: report on three cases. Leukemia 16:1871-1873

23. Takamatsu Y, Miyamoto T, Iwasaki H et al (1998) Remission induction by granulocyte colony stimulating factor in hypoplastic acute myelogenous leukemia complicated by infection. Acta Haematol 99: 224-230 